\title{
Neural network based geomagnetic estimation for multi-site observation system
}

\author{
Yuta Katori $^{\mathrm{a})}$ and Kan Okubo ${ }^{\text {b) }}$ \\ Graduate School of System Design, Tokyo Metropolitan University, \\ 6-6 Asahigaoka, Hino, Tokyo 191-0065, Japan \\ a)katori-yuta@ed.tmu.ac.jp \\ b) kanne@tmu.ac.jp
}

Abstract: For years, researchers have been studying the relation between earthquakes and geomagnetic field signals using observation results. Following our continuous geomagnetic observation, this report describes geomagnetic signal changes generated by earthquakes and tsunami waves. Results show that detection of their occurrence using geomagnetic field measurement is effective for providing an early alarm system for disaster mitigation. Robust detection requires the robust estimation of a geomagnetic field using multiple observation results. This study introduced a deep neural network (DNN) to estimate geomagnetic fields. Results clarified that the proposed DNN model using data of multiple axes at multiple observation points as input data provides efficient solutions for geomagnetic estimation.

Keywords: time-series geomagnetic estimation, anomaly detection, neural network, autoencoder, multi-site observation

Classification: Sensing

\section{References}

[1] M. J. S. Johnston, "Seismomagnetic observation with the July 8, 1986, ML 5.9 north palm springs earthquake," Science, vol. 237, no. 4819, pp. 1201-1203, Sept. 1987. DOI:10.1126/science.237.4819.1201

[2] K. Okubo, N. Takeuchi, M. Utsugi, K. Yumoto, and Y. Sasai, "Direct magnetic signals from earthquake rupturing: Iwate-Miyagi earthquake of M7.2, Japan," Earth Planet. Sci. Lett., vol. 305, no. 1-2, pp. 65-72, May 2011. DOI:10. 1016/j.eps1.2011.02.042

[3] K. Okubo, N. Takeuchi, M. Utsugi, K. Yumoto, and Y. Sasai, "Direct magnetic signals from earthquake faulting: Iwate-Miyagi earthquake of M 7.2, Japan," IAGA Sopron Assembly, Aug. 2009.

[4] K. Okubo, Y. Suyama, A. Takeuchi, T. Murai, T. Nagao, Y. Sasai, and N. Takeuchi, "Observation signals of earth's magnetic field: The 2011 off the Pacific coast of Tohoku earthquake of M 9.0, Japan," American Geophysical Union, Fall Meeting, NH23A-1544, Dec. 2011.

[5] G. E. Hinton and R. R. Salakhutdinov, "Reducing the dimensionality of data with neural networks," Science, vol. 313, no. 5786, pp. 504-507, July 2006. DOI:10.1126/science. 1127647

[6] P. Wessel, W. H. F. Smith, R. Scharroo, J. Luis, and F. Wobbe, "Generic mapping tools: Improved version released," Eos Trans. $A G U$, vol. 94, no. 45, 
pp. 409-410, Nov. 2013. DOI:10.1002/2013EO450001

[7] P. Wessel and W. H. F. Smith, "New, improved version of generic mapping tools released," Eos Trans. $A G U$, vol. 79, no. 47, p. 579, Nov. 1998. DOI:10. 1029/98EO00426

[8] P. Wessel and W. H. F. Smith, "New version of the generic mapping tools," Eos Trans. $A G U$, vol. 76, no. 33, p. 329, Aug. 1995. DOI:10.1029/95EO00198

[9] P. Wessel and W. H. F. Smith, "Free software helps map and display data," Eos Trans. $A G U$, vol. 72, no. 41, pp. 441-446, Oct. 1991. DOI:10.1029/ 90EO00319

[10] Kakioka Magnetic Observatory, "DIGITAL DATA SERVICE" Kakioka Magnetic Observatory, Japan Meteorological Agency, http://www.kakiokajma.go.jp/en/index.html, accessed Feb. 28, 2017.

[11] A. D. Chave, D. J. Thomson, and M. E. Ander, "On the robust estimation of power spectra, coherences, and transfer functions," J. Geophys. Res., vol. 92, no. B1, pp. 633-648, Jan. 1987. DOI:10.1029/JB092iB01p00633

[12] G. D. Egbert and J. R. Booker, "Robust estimation of geomagnetic transfer functions," Geophys. J. R. Astron. Soc., vol. 87, no. 1, pp. 173-194, Oct. 1986. DOI:10.1111/j.1365-246X.1986.tb04552.x

\section{Introduction}

\subsection{Background}

For years, researchers have been studying the relation between earthquakes and geomagnetic field signals using observation results [1]. Using data from our continuous geomagnetic observation, this report describes "co-faulting" geomagnetic signal changes generated when earthquakes occur [2].

Magnetic fields start changing almost simultaneously with the onset of the earthquake rupture before P-wave arrival [2]. This co-faulting geomagnetic phenomenon is useful for disaster prevention.

Recently, earthquake early warning systems, mainly by the Japan Meteorological Agency, have become operational in Japan. Nevertheless, people often receive a warning after the S-wave arrival. Making the system faster is extremely important for disaster reduction. Therefore, we have proposed adding an earthquake detection capability to usual detection systems by using magnetic field measurements with seismic wave measurements [3]. The geomagnetic field propagates at light speed. Therefore, earlier warning can be achieved using geomagnetic field measurements.

Moreover, giving an alarm when a tsunami occurs is important for disaster reduction. The large initial rise and fall of the sea surface associated with a tsunami increases or decreases the atmospheric pressure, which propagates to the ionosphere. Observation results demonstrate a geomagnetic field signal induced by this phenomenon [4]. Discrimination of geomagnetic field anomalies is very desirable to detect natural disasters and to give an efficacious alarm. Commonly, the relation between signals observed at multiple observation points is used for geomagnetic field estimation. However, no accurate geomagnetic field estimation method has been fully developed. Robust discrimination requires robust estimation of a geomagnetic field using multiple observation results. Therefore, we first introduce a deep neural network (DNN) for geomagnetic field estimation. 


\subsection{Observed geomagnetic signals}

Presumably, the estimated geomagnetic signals of two stations (points A and B) comprise local signals $\left(H_{A}^{(l)}, H_{B}^{(l)}\right)$ and a global signal, respectively, as $\left(H_{A}^{(g)}, H_{B}^{(g)}\right)$.

$$
\begin{aligned}
& H_{A}=H_{A}^{(g)}+H_{A}^{(l)} \\
& H_{B}=H_{B}^{(g)}+H_{B}^{(l)}
\end{aligned}
$$

Here, the local signal is obtained locally, whereas the global signal is observed simultaneously at multiple observation points induced by a common source. Therefore, some strong relation is expected to exist between $H_{A}^{(g)}$ and $H_{B}^{(g)}$.

Nevertheless, one cannot extract each local signal component by simple subtraction between signals obtained at two observation points because the signals induced by a common source are not the same.

$$
H_{A}-H_{B} \neq H_{A}^{(l)}
$$

Consequently, to detect $H_{A}^{(l)}$ or $H_{B}^{(l)}$, it is important to estimate $H_{A}^{(g)}$ or $H_{B}^{(g)}$ with high accuracy.

\section{Geomagnetic estimation}

\subsection{Autoencoder model}

For this study, we apply the autoencoder model, which is used as a deep learning technique [5]. Autoencoder is an artificial neural network typically used for dimensionality reduction. Autoencoder can reduce the computation costs of geomagnetic estimation, but it also provides noise reduction.

\subsection{Estimation model using autoencoder}

The neural network model is presented in Fig. 1. Here, we use GMT to produce a map of this figure $[6,7,8,9]$. As input data, we use geomagnetic field signals observed at three observation points (Memambetsu, Kakioka, Kanoya in Fig. 1) operated by the Japan Meteorological Agency.

Our model uses the three orthogonal vectors $(x, y, z)$ of geomagnetic fields at three stations as input values and the estimated vertical vector $(z)$ of geomagnetic field at IWK as output values. The I/O vectors of the neural network are assumed as a continuous time series of magnetic field data excluding DC the offset. The left part of the neural network schematic diagram in Fig. 1 is a pre-trained model of stacked encoder using the autoencoder model to extract the feature vector from the input. At right is a neural network model: a pre-trained stacked decoder model using autoencoder to rebuild a vector.

The center part (connecting part) of our model is a basic neural network model such as that presented in Fig. 1. The neural network part estimates the feature value of IWK from the input feature value of multiple axes at multiple observation points. Consequently, our model is assumed as a DNN model which is stacked by these three parts. Finally, fine tuning [5] is applied to our DNN model. The procedures for estimation from input data to IWK data using this model are summarized below.

1. Input a vector of time series of geomagnetic observation signal.

2. Calculate the feature extraction from input vector signals at each observation point. 
3. Estimate the extracted IWK feature from feature value at other points.

4. Rebuild the IWK vector from the IWK feature value.

In our model, input vectors are divided for each channel. Information compression is done for each channel. Therefore, transient noise of one channel can be prevented from affecting other channels. Moreover, by changing the input vector dimension ratio, one can increase the influence of the more related channel.

The dataset consist of $10 \mathrm{~Hz}$ geomagnetic data obtained at the geomagnetic observatory (MMB, KNY, KAK) [10] and geomagnetic data obtained at our observation point (IWK). Observation data obtained from Jan. 2015 to Dec. 2015 are used for neural network training. Datasets have 0.5 million data $(10 \mathrm{~Hz}$ sampled time series vector of 600 dimensions). We employ only observation data of 16:00-19:00 (UTC), which correspond to the middle of the night in Japan (i.e., low artificial noise period). Input data are normalized so that the vector of each dataset fits within the range of -1 to 1 .

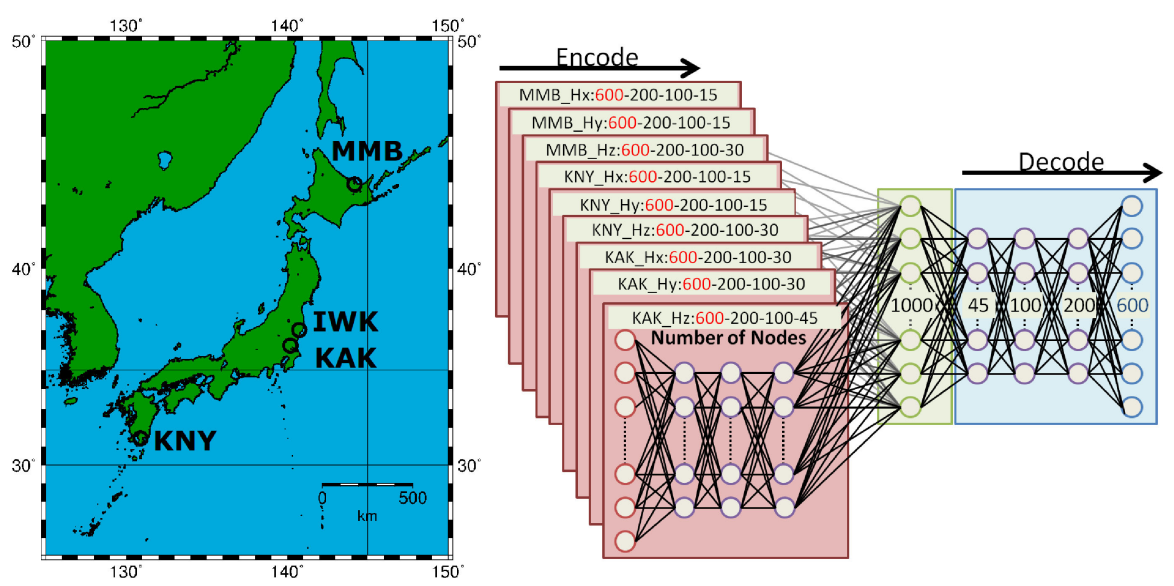

Fig. 1. Our DNN model for goemagnetic estimation.

\section{Results and discussion}

To estimate the geomagnetic field change, data observed in March 2016 (excluding the maintenance period) were used as test data. Regarding test data, we applied data of 16:00-19:00 UTC using a similar manner of training.

We compared some conditions of input data: (a) three observation points and three axes (DNN model), (b) three observation points and three axes (simple threelayer neural network model), and (c) three observation points and two axes $(x, z)$, (d) three observation points and one axis $(y, z)$, (e) three observation points and one axis (z), (f) two observation points (KAK, MMB) and $z$-axis, (g) one observation point (KAK) and the $z$-axis, (h) one observation point (KAK) and the $z$-axis (median smoothed transfer function), and (i) one observation point (KAK) and the $z$-axis (mean smoothed transfer function).

For (h) and (i), we also applied the mean filter or median filter to the result of the transfer function calculated for each frequency. For many researchers in the field of the geomagnetics, geomagnetic estimation using the transfer function has been reported as the usual method $[11,12]$. 
Fig. 2 presents the estimation results: left panels present those of 18:00-19:00 on March 7, 2016, whereas right panels present those of 18:00-19:00 on March 26, 2016. The respective K-index values in the KAK observatory were 3 and 1 on March 7 and March 26, where the K-index represents the local geomagnetic activity mainly in response to solar activity. Additionally, a geomagnetic storm occurred on March 26. Results were drawn by connecting $60 \mathrm{~s}$ short-term predictions. This figure depicts the results estimated under the conditions of (a), (b), (e), (g), and (h) are depicted. Additionally, signals observed at KAK and IWK are presented in Fig. 2.

Fig. 2 suggests that our DNN model using the data of three observation points and three axes as input data provides more similar results compared to the observed for one at IWK. Next, we quantitatively evaluated the estimation accuracy. The mean absolute error (MAE) was used as an evaluation value.

$$
M A E=\frac{1}{n} \sum_{i=0}^{n}\left|x_{i}-y_{i}\right|
$$

In this case, we calculated MAE between the estimation result and the result observed at IWK. Table I shows evaluation results obtained using MAE, which clarify that our DNN model using the data of three observation points and three axes as input data provides more reasonable and effective results than the observed values at IWK.
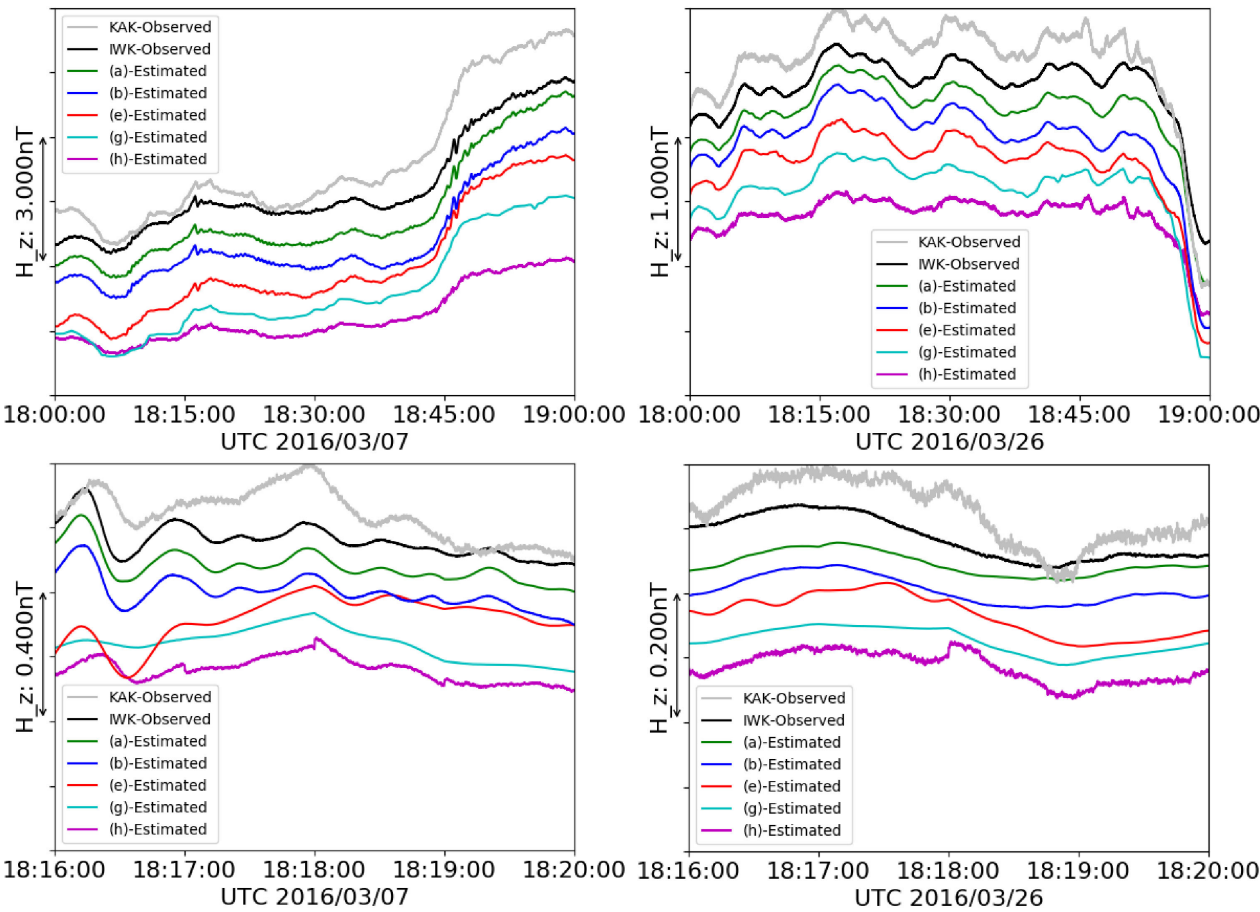

Fig. 2. Geomagnetic estimation results using our DNN model 
Table I. Mean absolute error of geomagnetic estimation

\begin{tabular}{lc}
\hline Estimation Model & MAE (pT) \\
\hline (a) Three observation points and three-axes (DNN model) & $\mathbf{0 . 0 0 6 4 2 0}$ \\
\hline (b) Three observation points and three-axis (simple NN model) & 0.006596 \\
\hline (c) Three observation points and $x$ and $z$ axes (DNN model) & 0.007588 \\
\hline (d) Three observation points and $y$ and $z$ axes (DNN model) & 0.009985 \\
\hline (e) Three observation points and $z$ axis (DNN model) & 0.011958 \\
\hline (f) Two observation points (KAK, MMB) and $z$ axis (DNN model) & 0.015829 \\
\hline (g) One observation point (KAK) and $z$ axis (DNN model) & 0.018546 \\
\hline (h) One observation point (KAK) and $z$ axis & 0.022231 \\
\hline (median smoothed transfer function) & \\
\hline (i) One observation point (KAK) and $z$ axis & 0.447584 \\
\hline
\end{tabular}

\section{Conclusion}

As described in this paper, we first reported a method of geomagnetic prediction using the deep neural network model. Results clarified that the proposed DNN model using data of multiple observation points and multiple axes as input data provides a more efficient solution for geomagnetic prediction. Results of this study suggest that the DNN model using multiple channels as input data provides accurate geomagnetic field estimation. Although the simple three-layer neural network yields precise results with similar accuracy, large computational cost is required for the three-layered neural network because it is a fully connected layer model. By contrast, no connection exists for each input channel in our DNN model. Therefore, its requirements for computational costs are smaller.

\section{Acknowledgement}

I would like to extend my greatest appreciation to Dr. Nobunao Takeuchi (Professor Emeritus, Akita Prefectural University), who provided helpful comments and suggestions. This study was partly supported by JSPS KAKENHI Grant Numbers 26289348, 24560507, and 23686130. 